\title{
Communication
}

\section{Proteomic and transcriptomic analysis of visual long-term memory in Drosophila melanogaster}

\author{
Huoqing Jiang ${ }^{1,2}$, Qinlong Hou ${ }^{1,2}$, Zhefeng Gong ${ }^{1 凶}$, Li Liu' ${ }^{1,3 凶}$ \\ ${ }^{1}$ State Key Laboratory of Brain and Cognitive Science, Institute of Biophysics, Chinese Academy of Sciences, Beijing 100101, China \\ ${ }^{2}$ Graduate University of the Chinese Academy of Sciences, Beijing 100039, China \\ ${ }^{3}$ Key Laboratory of Mental Health, Chinese Academy of Sciences, Beijing 100101, China \\ $\triangle$ Correspondence: zfgong@moon.ibp.ac.cn (Z. Gong), liuli@sun5.ibp.ac.cn (L. Liu) \\ Received January 25, 2011 Accepted February 27, 2011
}

\begin{abstract}
The fruit fly, Drosophila melanogaster, is able to discriminate visual landmarks and form visual long-term memory in a flight simulator. Studies focused on the molecular mechanism of long-term memory have shown that memory formation requires mRNA transcription and protein synthesis. However, little is known about the molecular mechanisms underlying the visual learning paradigm. The present study demonstrated that both spaced training procedure (STP) and consecutive training procedure (CTP) would induce long-term memory at 12 hour after training, and STP caused significantly higher 12-h memory scores compared with CTP. Labelfree quantification of liquid chromatography-tandem mass spectrometry (LC-MS/MS) and microarray were utilized to analyze proteomic and transcriptomic differences between the STP and CTP groups. Proteomic analysis revealed 30 up-regulated and 27 down-regulated proteins; Transcriptomic analysis revealed 145 up-regulated and 129 down-regulated genes. Among them, five candidate genes were verified by quantitative PCR, which revealed results similar to microarray. These results provide insight into the molecular components influencing visual long-term memory and facilitate further studies on the roles of identified genes in memory formation.
\end{abstract}

KEYWORDS visual learning and memory, Drosophila, long-term memory, microarray, liquid chromatography-tandem mass spectrometry

\section{INTRODUCTION}

For more than a century, at least two forms of memory have been distinguished by duration: short-term memory (STM), which is rapidly formed and can outlast training for minutes or hours, and long-term memory (LTM), which lasts from hours to days, weeks, or even years (Margulies et al., 2005). Behavioral and pharmacological evidences show that, in chicks and rats, multiple components of memory emerge at different times after training, and short-, middle-, and longterm memory phases exist (Rosenzweig et al., 1993). In Drosophila olfactory memory, behavioral and pharmacological experiments, as well as genetic analysis, have demonstrated that olfactory memory formation could be divided into four distinct phases: short-term memory (STM), middle-term memory (MTM), anesthesia-resistant memory (ARM), and long-term memory (LTM) (DeZazzo \& Tully, 1995; Margulies et al., 2005). In terms of visual memory in Drosophila, behavioral and pharmacological analyses have also provided clear evidences for four pharmacologically distinct memory phases after training: (1) a very short-term memory (preSTM), which lasts for approximately 2 min after training; (2) STM, which lasts approximately $20 \mathrm{~min}$; (3) ARM, which is present 20-120 min after training; and (4) LTM, which is activated at least $150 \mathrm{~min}$ after training and lasts for at least $48 \mathrm{~h}$ (Xia et al., 1997; Xia et al., 1998).

Identification and characterization of genes associated with memory formation have provided an abundance of information over the past decades, which extended the understanding of molecular mechanisms underlying this complex behavioral process (Davis and Squire, 1984; Mayford and Kandel, 1999; Hawkins et al., 2006). Studies utilizing a variety of learning paradigms in different species, including Aplysia, Drosophila, and rodents, have shown that specific genes regulating memory formation processes, through a variety of pathways, were involved in the molecular mechanisms of memory formation (Mayford and Kandel, 1999; Costa-Mattioli and Sonenberg, 2006; Keene and Waddell, 2007; Alberini, 2009). In long-term memory formation, a 
transcription factor, CAMP response element binding protein (CREB) was found to play a critical role. CREB can be activated by CAMP/PKA/CREB signaling pathway which is initiated by cAMP synthetase adenylyl cyclase (Yin et al., 1995). In addition, CREB can also be activated by mitogenactivated protein kinase/extracellular signal-regulated kinase (MAPK/ERK) pathway including mitogen-activated protein kinase, calmodulin and so on (Walton and Dragunow, 2000; Park and Cho, 2006). In Drosophila, many genes were found to be involved in olfactory memory: CAMP associated rutabaga and dunce related to STM, amnesiac related to MTM, and radish related to ARM (Margulies et al., 2005). Several genes were also found to specifically influence LTM, such as $n m d a$ (Xia et al., 2005), crammer (Comas et al., 2004), nf1 (Ho et al., 2007), notch (Ge et al., 2004; Presente et al., 2004), AKAP Yu (Lu et al., 2007), Klingon (Matsuno et al., 2009), and ben (Zhao et al., 2009). Moreover, microarray analysis was used to detect gene expression changes at 0,6 , and $24 \mathrm{~h}$ between spaced training and massed training groups. 42 candidate genes and the staufen/ pumilio pathway were found to relate to olfactory LTM in Drosophila (Dubnau et al., 2003).

Rutabaga and foraging were determined to be important for short-term visual pattern memory for Drosophila in a flight simulator (Liu et al., 2006; Wang et al., 2008a), dunce was necessary for STM, ARM, and LTM, and amnesiac for MTM (Gong et al., 1998). To date, however, little is known about the molecular-genetic mechanisms that contribute to visual LTM formation. The present study demonstrated that both spaced training procedure (STP) and consecutive training procedure (CTP) could induce long-term memory at 12 hours after training in visual learning paradigm. STP caused significantly higher 12-h memory scores compared with CTP in a flight simulator. Liquid chromatography-tandem mass spectrometry (LC-MS/MS) and microarray were utilized to compare proteomic and transcriptomic differences, respectively, between the two training procedures.

\section{RESULTS}

\section{STP induced significantly greater 12 -h memory scores compared with CTP}

To learn more about molecular mechanisms of visual longterm memory, flies were trained either with STP or CTP (Fig. 1; for details, see MATERIALS AND METHODS) in a flight simulator. During both STP and CTP training, performance indices (PIs) of the first training cycle were significantly less than those of the 2nd, 3rd, and last training cycles, and PIs of the second training cycle were also significantly less than those of the last training cycle (Fig. 2A). Moreover, there was no significant difference between PIs from the STP and CTP training during the 1st, 2nd, 3rd, and last training cycles (Fig. 2A).

Following STP or CTP training, flies were tested for immediate memory (0-h memory) and 12-h memory in a behavioral test procedure (Fig. 1C). 0 -h memory test consists of three 1-min test periods. Both 0-h STP and CTP groups showed significantly higher 0-h memory scores compared with zero. However, Pls for 0 -h memory were not significantly different between the 0-h STP and CTP groups (Fig. 2B). At 12 hours after training, flies from both groups were subject to a 1-min remind-training period and one test session consisting of three 1-min test periods (Fig. 1C). In the remind-training

A Spaced Training Procedure

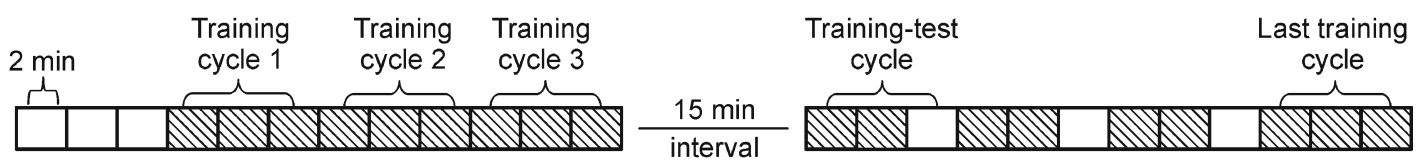

B

Consecutive Training Procedure

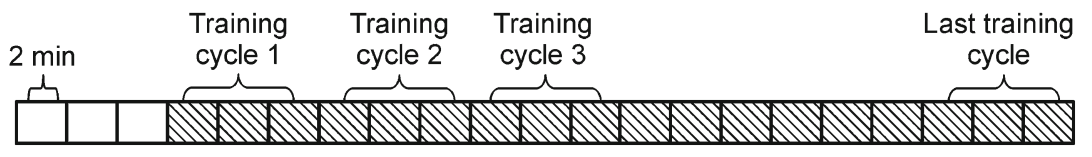

C

Behavioral Test Procedure

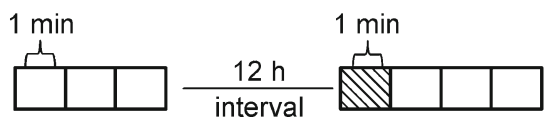

Test $\square \quad$ Training

Figure 1. The schematic diagram of spaced training procedure (STP; A), consecutive training procedure (CTP; B), and behavioral test procedure (C). 
A

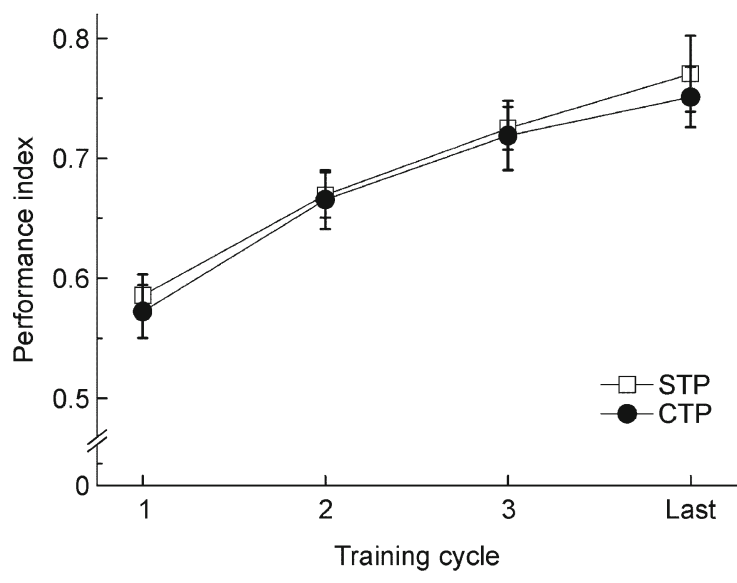

B

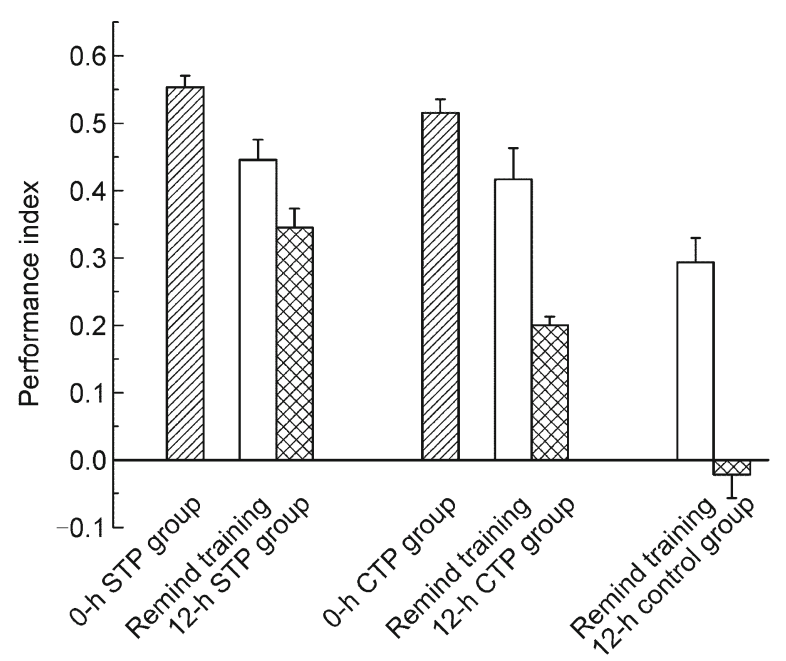

Figure 2. Spaced training procedure (STP) and consecutive training procedure (CTP) induced similar learning performances and immediate memory performances, but different 12-h memory performances. (A) learning performances of 12-h STP group $(n=10)$ and 12-h CTP group $(n=10)$ in the first, second, third, and last training cycles, calculated by averaging PIs of three 2-min training periods. The more training time was applied, the higher PI was achieved. In both STP and CTP groups, PIs of the first training cycle were significantly less than those of the $2 \mathrm{nd}$, 3rd, and last training cycles (STP group: $t=3.267, p<0.01$ between the first and 2nd 6-min training cycles; $t=5.597, p<00001$ between the first and 3rd; $t=5.107, p<0.001$ between the first and last. CTP group: $t=2.833, p<0.05$ between the first and 2 nd; $t=4.025, p<0.001$ between the first and 3rd; $t=5.31, p<0.001$ between first and last), and PIs of the second training cycle were also significantly less than those of the last (STP group: $t=2.738$, $p<0.05$; CTP group: $t=2.433, p<0.05$ ). Moreover, Pls of the $1 \mathrm{st}, 2 \mathrm{nd}, 3 \mathrm{rd}$, and last training cycles showed no significant difference between the STP and CTP groups. (B) Although both STP and CTP groups showed significantly higher 0-h memory scores compared with zero (STP group: $t=33.42, p<0.001$; CTP group: $t=24.19, p<0.001$ ), 0 -h memory scores were not significantly different between the STP and CTP groups $(t=1.401, p>0.05)$. The same as remind-training in 12 hours after training, Pls of the STP group were not significantly different from the CTP group $(t=0.519, p>0.05)$. However, 12-h memory scores of both groups were significantly higher than zero (STP: $t=12.14, p<0.001$; CTP: $t=16.34, p<0.001$ ), and the 12-h memory scores following STP training were significantly greater than that after CTP training $(t=4.68, p<0.001)$. The control group $(n=10)$ in which flies flew in flight simulators for two 24 min sessions and one $15 \mathrm{~min}$ interval in between without any training showed no $12-\mathrm{h}$ memory $(-0.022 \pm$ $0.035, t=0.62461, p=0.5477$ compared to zero), although the $\mathrm{Pl}$ of remind-training was significantly higher than zero $(0.293 \pm$ $0.036, t=8.10, p<0.001)$.

period, Pls of the 12-h STP group were not significantly different from 12-h CTP group (Fig. 2B). However, Pls for 12-h memory of 12-h STP group were significantly greater than that of 12-h CTP group, despite that both Pls were significantly higher than zero (Fig. 2B). One control group was "trained" with STP, but all training periods were changed to test periods. It meant that these flies flew in flight simulators for two $24 \mathrm{~min}$ sessions with one $15 \mathrm{~min}$ interval in between and without any training. After 12 hours, these flies received a 1-min remind-training period and one test session consisting of three 1-min test periods, and results showed that no memory was induced, although the $\mathrm{PI}$ from remind-training was significantly higher than zero (Fig. $2 \mathrm{~B}$ ).

These results demonstrated that both STP and CTP training could induce immediate memory and long-term memory in visual learning paradigm. There was no significant difference between 0-h memory scores of the STP and CTP groups, but the STP group showed significantly higher 12-h memory scores than the CTP group.

\section{Proteomic profiling of differentially expressed proteins between the STP and CTP groups}

In our behavioral test, both STP and CTP could induce LTM in 12 hours in the visual learning paradigm while the STP group showed significantly higher 12-h memory scores than the CTP group. Such a difference might involve variations at molecular level, which underlie the formation of LTM induced by STP but not by CTP.

In general, proteins are considered direct effectors in biological functions. The label-free quantitative test of LCMS/MS was first used to analyze proteomic changes between the 12-h STP and 12-h CTP groups. 50 flies were trained by CTP, and another 50 flies were trained by STP. After 12 hours, the flies were put in liquid nitrogen. The heads were used to generate protein extracts. Whole protein was used for 1-D LCMS/MS analysis, and the data were analyzed by Decyder-MS.

A total of 57 proteins exhibited a significant difference between the 12-h STP and 12-h CTP groups $(p<0.05)$ : 30 
proteins were up-regulated and 27 were down-regulated (Fig. S1). These proteins may be responsible not only for the formation of LTM but also for the increment in LTM induced by STP compared to that induced by CTP.

\section{Transcriptional profiling of differentially expressed genes for long-term memory in the STP and CTP groups}

In addition to proteomic changes, we also investigated differences of gene expression between 12-h STP and 12-h CTP groups using DNA microarray. For microarray analysis, 90 flies were trained using STP, and another 90 were trained with CTP. These flies were sacrificed 12 hours after training, and their heads were stored in liquid nitrogen immediately. We called them 12-h STP group and 12-h CTP group. The heads from 12-h STP group and 12-h CTP group were divided into three sets, respectively, to generate three independent RNA extracts. Data derived from replicates ( $n$ = 3) in the 12-h STP and 12-h CTP groups were used to perform pairwise comparisons. Unpaired $t$-test was used to determine significantly altered gene expression $(p<0.05)$. A total of 274 genes exhibited significant changes: 145 were upregulated and 129 were down-regulated in the 12-h STP group, compared with the 12-h CTP group (Fig. S2). These results revealed LTM-related genes were differentially transcribed between STP and CTP. Among the 145 up-regulated genes, some genes which were involved in cAMP/PKA/ CREB or MAPK/ERK pathway showed significantly higher expression, such as ac3 (adenylate cyclase 3), mkp3 (mitogen-activated protein kinase phosphatase 3), p38c (MAP kinase activity), cam (calmodulin), and strn-mlck (calmodulin-dependent protein kinase activity).

To evaluate the reliability of up-regulation and downregulation of genes in comparison between 12-h STP and 12-h CTP groups, we chose five genes for quantitative PCR
(qPCR): the genes strn-mlck and $p 38 c$ are involved in MAPK/ ERK pathway, and the genes $m t h / 2$ and $t / d$ were reported to be involved in axon guidance and synaptic transmission (Aberle et al., 2002; Mazzucchelli et al., 2002; Kelly et al., 2003; Keshishian and Kim, 2004; Sharma and Carew., 2004; Ho et al., 2007), and an unknown gene CG1673. For qPCR experiments, 150 flies were trained by STP, and another 150 were trained by CTP. After training, they were kept for 12 hours and sacrificed. Their heads were stored in liquid nitrogen immediately. The heads from 12-h STP group and 12-h CTP group were divided into five sets, respectively, to generate five independent RNA extracts for qPCR experiments. The qPCR results showed that the tendency of these five genes which were up-regulated or down-regulated were consistent with microarray results (Fig. 3).

\section{DISCUSSION}

The present behavioral experiments have demonstrated that 12-h visual long-term memory can be detected following both STP and CTP training procedures. However, the different training procedures induced different 12-h memory scores. STP induced significantly greater $12-\mathrm{h}$ memory scores compared with CTP. Proteomic and transcriptomic changes were compared between the 12-h STP and 12-h CTP groups. In total, 57 proteins were changed at proteomic level and 274 genes were changed at transcriptomic level.

In visual learning paradigm, a single fly was trained for 36 min and after 12 hours the visual long-term memory could be detected after STP or CTP training. In the 12-h control group, the $36 \mathrm{~min}$ training was replaced by test mode. The flies flew in flight simulators for the same time as those in the STP group, but visual long-term memory could not be induced. Proteomic changes were compared between the 12-h STP and 12-h CTP groups and 57 proteins were

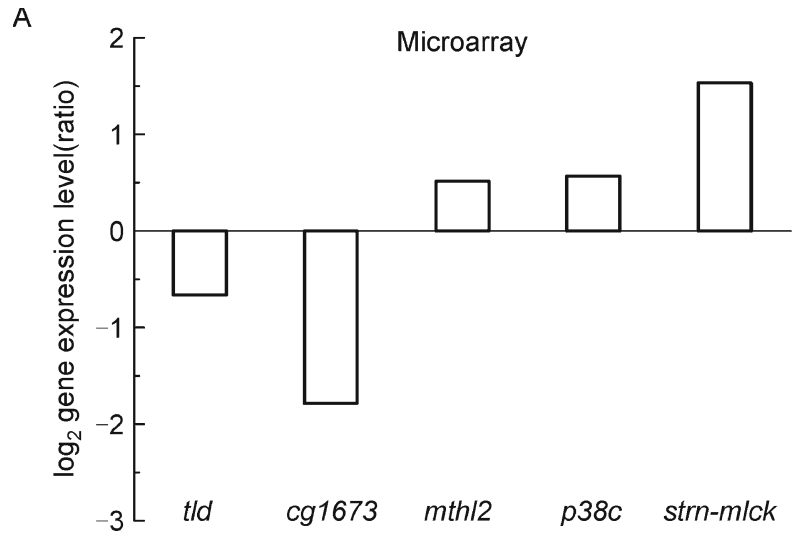

B

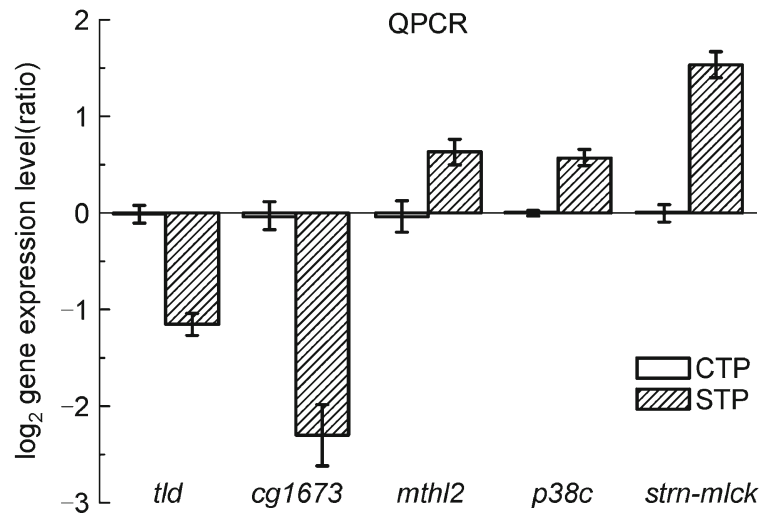

Figure 3. Expressional profile of five genes were consistent in microarray and qPCR. (A) The gene expression ratios of 12-h STP group to the 12-h CTP group for five genes from microarray analysis are shown. (B) The relative expression levels of the five genes in the 12-h CTP group and 12-h STP group from qPCR experiments. Results of qPCR were expressed as mean \pm SE, $n=5$ for each group. STP, spaced training procedure; CTP, consecutive training procedure. 
obtained. All proteins were classified by their gene ontology (GO) function. Most of them belonged to the categories such as molecular binding, metabolism, cell communication, signal transduction and cell organization. For example, CG1695, as a signal transduction factor, has been reported to be a Rab GTPase activator, which is involved in specificity of vesicle targeting. Therefore, it is possible that CG1695 influences memory through regulating vesicle transport. The protein $\mathrm{Cib}$ belongs to cell organization. The cib has been shown to influence fly ellipsoid body structure (Boquet et al., 2000), which is known to be involved in visual and olfactory memory (Wu et al., 2007; Wang et al., 2008a). Unexpectedly, one important transcription factor CREB which plays important roles in LTM formation was not detected. This could be due to the following reasons: (1) in the present study, both SCP and CTP groups exhibited LTM, although there were performance differences. CREB might have been activated in both groups, thereby resulting in no expressional differences. (2) the entire brain was used for high-throughput experiments, so that regional differences in gene expression could not be detected.

We compared the differences at transcriptional level between the 12-h STP group and 12-h CTP group, and there were 274 candidates determined by microarray analysis. Classified by their GO function, some of them were involved in biological processes that might be related to long-term memory, such as signal transduction, transcription/ translation, synaptic plasticity, and post-translational protein modification. Some genes in CAMP/PKA/CREB or MAK/ERK signaling pathway showed significantly higher expression level in STP than in CTP, which could be the reasons underlying different 12-h memory scores in the different training procedures. For example, in the post-translational protein modification category, P38c was identified by Interpro as a homologue of P38a and P38b (P38 mitogen-activated protein kinase, MAPK). It is thought that MAPK influences memory by activating transcriptional factors and inducing synthesis of new transcripts and proteins. In Aplysia, MAPK was reported to regulate sensory-motor synaptic plasticity, and was shown to be involved in sensitization memory (Sharma and Carew, 2004). In rats, an injection of MAPK/ ERK blocked LTM (Kelly et al., 2003), and knocking-out ERK1 in the mouse enhanced synaptic plasticity and memory (Mazzucchelli et al., 2002). In Drosophila, NF1 (Neurofibromin 1) was reported to play a role in LTM through the Ras/ MAPK pathway (Ho et al., 2007). These results suggest that P38c may be involved in LTM through the MAPK/ERK pathway. Strn-mlck encodes a myosin light-chain kinase. In mice, myosin $\mathrm{Vb}, \mathrm{a} \mathrm{Ca}^{2+}$-sensitive motor protein, conducts spine trafficking during long-term potentiation (LTP) of synaptic strength (Wang et al., 2008b). Thus, Strn-m/ck is likely to regulate memory formation by recycling endosomes in Drosophila.

Compared with proteomic results, the analysis revealed that only few genes (Gpdh and CG12288) were found in the transcriptomic studies. The possible reasons include the procedure difference in sample preparation between genomics and proteomics and, the technical limitation of proteomics, as well as the delay between transcription and translation. In brief, this study provides insight into the molecular components that influence visual long-term memory and serves to elucidate the roles of identified genes in memory formation.

\section{MATERIALS AND METHODS}

\section{Flies}

Three- to four-day-old wild type Canton- $S$ flies were used throughout this study. Flies were raised at $(24 \pm 1)^{\circ} \mathrm{C}$ in a $12: 12 \mathrm{~h} \mathrm{light/dark}$ cycle, with the light cycle beginning at $7 \mathrm{am}$. Flies were fed on standard corn meal/molasses food medium (Guo et al., 1996).

\section{Learning apparatus}

Visual learning experiments were performed in a flight simulator. For apparatus, preparation and handling of flies, details can be seen in previously described methods (Heisenberg and Wolf, 1984; Wolf and Heisenberg, 1991). For the learning paradigm, procedure, and definition of performance index (PI), data can be seen in the literature (Wang et al., 2008a). The reinforcer was an adjustable infrared laser (wavelength, $10.6 \mu \mathrm{m}$; power, $300 \mathrm{~mW}$ ), which was directed from above at the fly abdomen and was computer-controlled. The laser spot diameter was $0.4 \mathrm{~mm}$, and the laser beam pulsed with $50-\mathrm{ms}$ duration and 50 -ms interval.

\section{Spaced training procedure}

The spaced training procedure (STP; Fig. 1A) included: pre-training session, massed training session, spaced training session, and test session. The pre-training session comprised three consecutive 2-min test periods without heat reinforcement. The massed training session was composed of three consecutive 6-min training cycles, which were subdivided into three 2-min training periods. The flies were tested for spontaneous pattern preference during the pre-training session, and were trained to avoid heat-associated patterns during the training cycles.

Subsequently, a 15-min interval took place, during which the torque meter and fly were lifted above the panorama. The fly was then lowered to the center of the panorama for approximately $2 \mathrm{~min}$ before the spaced training session began. This session consisted of three training-test cycles and one 6-min training cycle. One training-test cycle comprised two 2-min training periods and one 2-min test period.

\section{Consecutive training procedure}

The consecutive training procedure (CTP; Fig. 1B) included: pretraining session and massed training session. The pre-training session was composed of three consecutive 2-min test periods. The massed training session comprised six consecutive 6-min training cycles, which were subdivided into three 2-min training periods. 


\section{Behavioral test procedure}

The behavioral test procedure (Fig. 1C) included immediate memory (0-h memory) and12-h memory tests. Immediately following STP or CTP, the fly was tested for 0-h memory in one test session of three 1-min test periods. The fly was allowed to make a choice between the pattern types without being heated. Prior to testing, the panorama was set to a new, random position. After 0-h memory test, the fly was removed from the torque meter and placed into the chamber. In 12 hours, and approximately 10 min prior to testing, the fly was fixed to the torque meter. At 3 min prior to testing, the fly was placed in the center of the panorama, where it remained for $2 \mathrm{~min}$. The fly was then trained with 1-min remind training, and was tested with one test session consisting of three 1-min test periods between the upright $\mathrm{T}$ and inverted T. Prior to testing, the panorama was set to a new, random position. The flies which went through the behavioral test for memory were not included in the microarray experiments.

In both STP and CTP groups, Pls of the first, second, third, and last training cycles were calculated by averaging the three Pls from the training periods, and Pls of 0-h or 12-h memory were calculated by averaging PIs from the three 1-min test periods. For statistics, twosample unpaired $t$-test was used to assess unplanned pairwise comparisons between group means.

\section{Label-free quantification LC-MS/MS}

\section{Protein extraction and digestion}

At 12 hours after STP or CTP training, the fly heads were removed and placed in liquid nitrogen. A total of 50 heads per group were homogenized in detergent-free lysis buffer $(150 \mathrm{mmol} / \mathrm{L}$ Tris, $\mathrm{pH} 8.5 /$ $10 \mathrm{mmol} / \mathrm{L} \mathrm{DTT} / 0.1 \mathrm{mmol} / \mathrm{L}$ cocktail $/ 8 \mathrm{mmol} / \mathrm{L}$ urea), separately, on ice. Lysates were homogenized by supersonic ultrasound $(80 \mathrm{~W}$, five times, $9 \mathrm{~s}$ each, $15 \mathrm{~s} /$ interval), and then centrifuged at $15,000 \mathrm{~g}$ for $45 \mathrm{~min}$ at $4^{\circ} \mathrm{C}$ to remove debris. The supernatants were transferred to separate tubes, and protein concentrations were determined using the Bradford assay (Bradford, 1976; Stoscheck, 1990). The following "shotgun" and "label-free detection and peptide quantification" were performed by Shanghai Applied Protein Technology Co. Ltd.

\section{Shotgun}

The precipitate was dissolved in lysis buffer (8 mol/L urea, 4\% 3-[(3cholamidopropyl)dimethylammonio]-1-propanesulfonate (CHAPS), $40 \mathrm{mmol} / \mathrm{L}$ Tris, $65 \mathrm{mmol} / \mathrm{L}$ dithiothreitol (DTT)) and was quantified using the Bradford assay. Then, $0.1 \%$ acetic acid, $50 \%$ acetone, and $50 \%$ ethanol $(v / v 1: 5)$ were added to the mixture, which was incubated at $-20^{\circ} \mathrm{C}$ overnight. The supernatant was removed by centrifugation at 14,000 rotation $/ \mathrm{min}$ for $40 \mathrm{~min}$ at $4^{\circ} \mathrm{C}$. The precipitate was dissolved in $100 \mu \mathrm{L}$ buffer $(6 \mathrm{~mol} / \mathrm{L}$ guanidine hydrochloride, $100 \mathrm{mmol} / \mathrm{L}$ ammonium bicarbonate, $\mathrm{pH}$ 8.5) and mixed with $1 \mu \mathrm{L}$ $1 \mathrm{~mol} / \mathrm{L}$ DTT. The mixture was incubated at $37^{\circ} \mathrm{C}$ for $30 \mathrm{~min}$, and then $2.5 \mu \mathrm{L} 1 \mathrm{~mol} / \mathrm{L}$ iodoacetamide (IAA) was added and incubated for an additional $30 \mathrm{~min}$ at room temperature in the dark. The sample was exchanged with $100 \mathrm{mmol} / \mathrm{L}$ ammonium bicarbonate buffer, $\mathrm{pH} 8.5$, followed by incubation with trypsin $(50: 1)$ at $37^{\circ} \mathrm{C}$ overnight.

1-D LC-MS/MS chromatography was performed using the Ettan ${ }^{\mathrm{TM}}$ MDLC system (GE Healthcare). The samples were de-salted on RP trap columns (Zorbax 300 SB C18, Agilent Technologies), and then separated on a C18 reverse-phase column (RP, $180 \mu \mathrm{m} \times 150 \mathrm{~mm}$, BioBasic ${ }^{\circledR}$ C18, $5 \mu \mathrm{m}$, Thermo Hypersil-Keystone). The pump flow rate was split at $1: 100$ for a column flow rate of $1.5 \mu \mathrm{L} / \mathrm{min}$. The column effluent was directly electrosprayed using the orthogonal metal needle source without further splitting. Mobile phase A was $0.1 \%$ formic acid in water, and mobile phase $B$ was $0.1 \%$ formic acid in acetonitrile. The separation of peptides obtained by enzymatic digestion of bile samples was achieved with a gradient of $2 \%-80 \% \mathrm{~B}$ over $600 \mathrm{~min}$. The column effluent from the reverse-phase column was analyzed with an A Finnigan ${ }^{\mathrm{TM}} \mathrm{LTQ}^{\mathrm{TM}}$ ion-trap mass spectrometer. The micro-electrospray interface used a $30-\mu \mathrm{m}$ metal needle, which was orthogonal to the linear ion trap quadrupole (LTQ) inlet. The mass spectrometer was set to one full MS scan, followed by ten MS/MS scans on the three most intense ions from the MS spectra, with the following Dynamic Exclusion ${ }^{\mathrm{TM}}$ settings: repeat count, 1; repeat duration, $0.5 \mathrm{~min}$; exclusion duration, $3.0 \mathrm{~min}$.

\section{Label-free detection and peptide quantification}

Peptide detection, elution profile comparison, background subtraction, and peptide quantification were performed using a full-scan precursor mass spectrum in fully automatic mode using DeCyder MS software version 1.0 (GE Healthcare). The presentation of LC-MS spectra as 2-D signal intensity maps was used for visual raw data quality assessment. The PepDetect module of the software was used for automated peptide detection, charge state assignments based on resolved isotopic peaks and consistent spacing between consecutive charge states, and quantification based on MS signal intensities of individual LC-MS analyses. The final step consisted of matching peptides across different signal intensity maps using the PepMatch module, which resulted in a quantitative comparison. The intensity distributions for all peptides detected in each sample were used for normalization (no internal standards were added to the samples), and peptides were identified by importing TurboSEQUEST search results into the Pep-Match module. The criteria for Sequest score are: Xcorr $\epsilon$ 1.9 for Charge +1 ; Xcorr $\epsilon 2.2$ for Charge +2 ; and Xcorr $\epsilon 3.75$ for Charge +3 . The next step involved matching of peptides that fell within a user-defined mass and retention time interval in a quantitative comparison across different signal intensity maps from replicate analyses in the PepMatch module.

$P$ value is calculated by $t$-test comparison between ion intensity of CTP repeats and ion intensity of STP repeats, both of which were three times.

\section{DNA microarray chip}

The GeneChip ${ }^{\circledR}$ Drosophila Genome 2.0 Array (Affymetrix) was used in this study. The flies used for Gene Chip analysis were trained either with STP or CTP alternately in the flight simulator. Without behavioral test procedure, the fly heads were removed and stored with liquid nitrogen in cryo tubes (one fly head per tube) at 0 or 12 hour after STP or CTP training. After collecting enough flies for each group, total cellular RNA was isolated using Trizol reagent (Invitrogen Inc.).

The samples of total RNA were sent to a company (Shanghai Biochip Co., Ltd.) for array experiments and data analysis. In brief, biotinylated cRNA probes for Affymetrix chip hybridization were generated according to Affymetrix protocols. Reverse transcription was performed with anchored oligo-DT primers containing a T7 RNA 
polymerase sequence. cRNA was resuspended in $200 \mu \mathrm{L}$ hybridization solution. Hybridization reactions, labeling, and chip scanning were performed according to Affymetrix protocols.

The experiments were done once with total RNA extracted from either 0-h STP or 0-h CTP groups. However, the experiments were repeated three times with different batches of total RNA extracted from either 12-h STP or 12-h CTP groups. To analyze the array results between 12-h STP and 12-h CTP groups, MAS5.0 software was used for normalization and background correction with the algorithm RMA (Robust Multichip Averaging). Then, baseline transform was used to change baseline to median of all samples: for each probe the median of the log summarized values from all the samples is calculated and subtracted from each of the samples. In the end, unpaired $t$-test was used for selecting the different genes $(p<0.05)$. Baseline transformation and unpaired $t$-test was carried out with Genespring 9.0 software.

\section{Quantitative PCR}

qPCR was used in a standard way. In brief, $0.5 \mu \mathrm{L}$ of cDNA mixture, prepared from about 30 fly heads at 12 hours after STP or CTP training, were used as templates and tested on a Chromo 4 system (MJ Research/Bio-Rad). For each group, cDNA was obtained from five independent RNA preparations for repeating and averaging. The relative differences in mRNA expression levels between the STP and CTP groups were quantified by comparing expression levels to standard curves. The curves were plotted using corresponding recombinant plasmids, and were normalized to expression levels of actin. Unpaired $t$-test was used for statistical analysis of relative mRNA levels. The primers used for qPCR were as follows: qPCRactin upper, 5'-CAGGCGGTGCTTTCTCTCTA-3'; qPCR-actin lower, 5'-AGCTGTAACCGCGCTCAGTA-3'; qPCR-CG1673 upper, 5'CTACGCACCCACAATCAATG-3'; qPCR-CG1673 lower, 5'ATACCGGGCAGGATTAGACC-3'; qPCR-Strn-mlck-1 upper, 5'GCGATGAATCTGAACCCATT-3'; qPCR-Strn-mlck-1 lower, 5'GTTGATGATCAGCTCGCAAA-3'; qPCR-tld upper, 5'-CAAGCCGCCAATCATAATCT-3'; qPCR-tld lower, 5'-AGTATGCGCACCTCGCTATT-3'; qPCR-p38c upper, 5'-TACTCGCGTTCGAAAAGGAT-3'; qPCR-p38c lower, 5'-AGGACTTTCCGATTGTGTGG-3'; qPCR$m t h / 2$ upper, 5'-GCCCTGCGATGATATGTTTT-3'; qPCR- $m$ th/2 lower, 5'-GTGGGGTATAATGCGAATGG-3'.

\section{ACKNOWLEDGEMENTS}

We thank Shanghai Applied Protein Technology Co. Ltd. for label-free quantification of LC-MS/MS experiments and data analysis. We also thank Haiyun Gong for technical assistance. This work was supported by the National Basic Research Program of China (the 973 Program) (Grant No. 2009CB918702), the National Natural Science Foundation of China (Grant Nos. 30921064, 30625022, 31030037 and 31070944), and the External Cooperation Program of the Chinese Academy of Sciences (Grant No. GJHZ1005).

Supplementary material is available in the online version of this article at http://dx.doi.org/10.1007/s13238-011-1019-0 and is accessible for authorized users.

\section{ABBREVIATIONS}

ARM: anesthesia-resistant memory; CTP: consecutive training procedure; GO: gene ontology; LC-MS/MS: liquid chromatography- tandem mass spectrometry; LTM: long term memory; MTM: middleterm memory; PI: performance index; STM: short-term memory; STP: spaced training procedure; LTP: long term potentiation

\section{REFERENCES}

Aberle, H., Haghighi, A.P., Fetter, R.D., McCabe, B.D., Magalhães, T. R., and Goodman, C.S. (2002). wishful thinking encodes a BMP type II receptor that regulates synaptic growth in Drosophila. Neuron 33, 545-558.

Alberini, C.M. (2009). Transcription factors in long-term memory and synaptic plasticity. Physiol Rev 89, 121-145.

Boquet, I., Boujemaa, R., Carlier, M.F., and Préat, T. (2000). Ciboulot regulates actin assembly during Drosophila brain metamorphosis. Cell 102, 797-808.

Bradford, M.M. (1976). A rapid and sensitive method for the quantitation of microgram quantities of protein utilizing the principle of protein-dye binding. Anal Biochem 72, 248-254.

Comas, D., Petit, F., and Preat, T. (2004). Drosophila long-term memory formation involves regulation of cathepsin activity. Nature 430, 460-463.

Costa-Mattioli, M., and Sonenberg, N. (2006). Translational control of long-term synaptic plasticity and memory storage by elF2alpha. Crit Rev Neurobiol 18, 187-195.

Davis, H.P., and Squire, L.R. (1984). Protein synthesis and memory: a review. Psychol Bull 96, 518-559.

DeZazzo, J., and Tully, T. (1995). Dissection of memory formation: from behavioral pharmacology to molecular genetics. Trends Neurosci 18, 212-218.

Dubnau, J., Chiang, A.S., Grady, L., Barditch, J., Gossweiler, S., McNeil, J., Smith, P., Buldoc, F., Scott, R., Certa, U., et al. (2003). The staufen/pumilio pathway is involved in Drosophila long-term memory. Curr Biol 13, 286-296.

Ge, X., Hannan, F., Xie, Z., Feng, C., Tully, T., Zhou, H., Xie, Z., and Zhong, Y. (2004). Notch signaling in Drosophila long-term memory formation. Proc Natl Acad Sci U S A 101, 10172-10176.

Gong, Z., Xia, S., Liu, L., Feng, C., and Guo, A. (1998). Operant visual learning and memory in Drosophila mutants dunce, amnesiac and radish. J Insect Physiol 44, 1149-1158.

Guo, A., Li, L., Xia, S.Z., Feng, C.H., Wolf, R., and Heisenberg, M. (1996). Conditioned visual flight orientation in Drosophila: dependence on age, practice, and diet. Learn Mem 3, 49-59.

Hawkins, R.D., Kandel, E.R., and Bailey, C.H. (2006). Molecular mechanisms of memory storage in Aplysia. Biol Bull 210, 174-191.

Heisenberg, M., and Wolf, R. (1984). Vision in Drosophila: genetics of microbehavior. Berlin; New York: Springer-Verlag.

Ho, I.S., Hannan, F., Guo, H.F., Hakker, I., and Zhong, Y. (2007). Distinct functional domains of neurofibromatosis type 1 regulate immediate versus long-term memory formation. J Neurosci 27, 6852-6857.

Keene, A.C., and Waddell, S. (2007). Drosophila olfactory memory: single genes to complex neural circuits. Nat Rev Neurosci 8, 341-354.

Kelly, A., Laroche, S., and Davis, S. (2003). Activation of mitogenactivated protein kinase/extracellular signal-regulated kinase in hippocampal circuitry is required for consolidation and reconsolidation of recognition memory. J Neurosci 23, 5354-5360.

Keshishian, H., and Kim, Y.S. (2004). Orchestrating development and function: retrograde BMP signaling in the Drosophila nervous 
system. Trends Neurosci 27, 143-147.

Liu, G., Seiler, H., Wen, A., Zars, T., Ito, K., Wolf, R., Heisenberg, M., and Liu, L. (2006). Distinct memory traces for two visual features in the Drosophila brain. Nature 439, 551-556.

Lu, Y., Lu, Y.S., Shuai, Y., Feng, C., Tully, T., Xie, Z., Zhong, Y., and Zhou, H.M. (2007). The AKAP Yu is required for olfactory long-term memory formation in Drosophila. Proc Natl Acad Sci U S A 104, 13792-13797.

Margulies, C., Tully, T., and Dubnau, J. (2005). Deconstructing memory in Drosophila. Curr Biol 15, R700-R713.

Matsuno, M., Horiuchi, J., Tully, T., and Saitoe, M. (2009). The Drosophila cell adhesion molecule klingon is required for long-term memory formation and is regulated by Notch. Proc Natl Acad Sci U S A 106, 310-315.

Mayford, M., and Kandel, E.R. (1999). Genetic approaches to memory storage. Trends Genet 15, 463-470.

Mazzucchelli, C., Vantaggiato, C., Ciamei, A., Fasano, S., Pakhotin, P., Krezel, W., Welzl, H., Wolfer, D.P., Pagès, G., Valverde, O., et al. (2002). Knockout of ERK1 MAP kinase enhances synaptic plasticity in the striatum and facilitates striatal-mediated learning and memory. Neuron 34, 807-820.

Park, E.M., and Cho, S. (2006). Enhanced ERK dependent CREB activation reduces apoptosis in staurosporine-treated human neuroblastoma SK-N-BE(2)C cells. Neurosci Lett 402, 190-194.

Presente, A., Boyles, R.S., Serway, C.N., de Belle, J.S., and Andres, A.J. (2004). Notch is required for long-term memory in Drosophila. Proc Natl Acad Sci U S A 101, 1764-1768.

Rosenzweig, M.R., Bennett, E.L., Colombo, P.J., Lee, D.W., and Serrano, P.A. (1993). Short-term, intermediate-term, and longterm memories. Behav Brain Res 57, 193-198.

Sharma, S.K., and Carew, T.J. (2004). The roles of MAPK cascades in synaptic plasticity and memory in Aplysia: facilitatory effects and inhibitory constraints. Learn Mem 11, 373-378.

Stoscheck, C.M. (1990). Quantitation of protein. Methods Enzymol 182, 50-68.

Walton, M.R., and Dragunow, I. (2000). Is CREB a key to neuronal survival? Trends Neurosci 23, 48-53.

Wang, Z., Edwards, J.G., Riley, N., Provance, D.W. Jr, Karcher, R., Li, X.D., Davison, I.G., Ikebe, M., Mercer, J.A., Kauer, J.A., et al. (2008b). Myosin Vb mobilizes recycling endosomes and AMPA receptors for postsynaptic plasticity. Cell 135, 535-548.

Wang, Z., Pan, Y., Li, W., Jiang, H., Chatzimanolis, L., Chang, J., Gong, Z., and Liu, L. (2008a). Visual pattern memory requires foraging function in the central complex of Drosophila. Learn Mem 15, 133-142.

Wolf, R., and Heisenberg, M. (1991). Basic organization of operant behavior as revealed in Drosophila flight orientation. J Comp Physiol A 169, 699-705.

Wu, C.L., Xia, S., Fu, T.F., Wang, H., Chen, Y.H., Leong, D., Chiang, A.S., and Tully, T. (2007). Specific requirement of NMDA receptors for long-term memory consolidation in Drosophila ellipsoid body. Nat Neurosci 10, 1578-1586.

Xia, S., Liu, L., Feng, C., and Guo, A. (1997). Memory consolidation in Drosophila operant visual learning. Learn Mem 4, 205-218.

Xia, S., Miyashita, T., Fu, T.F., Lin, W.Y., Wu, C.L., Pyzocha, L., Lin, I. R., Saitoe, M., Tully, T., and Chiang, A.S. (2005). NMDA receptors mediate olfactory learning and memory in Drosophila. Curr Biol 15, 603-615.

Xia, S.Z., Feng, C.H., and Guo, A.K. (1998). Multiple-phase model of memory consolidation confirmed by behavioral and pharmacological analyses of operant conditioning in Drosophila. Pharmacol Biochem Behav 60, 809-816.

Yin, J.C., Wallach, J.S., Wilder, E.L., Klingensmith, J., Dang, D., Perrimon, N., Zhou, H., Tully, T., and Quinn, W.G. (1995). A Drosophila CREB/CREM homolog encodes multiple isoforms, including a cyclic AMP-dependent protein kinase-responsive transcriptional activator and antagonist. Mol Cell Biol 15, 5123-5130.

Zhao, H., Zheng, X., Yuan, X., Wang, L., Wang, X., Zhong, Y., Xie, Z., and Tully, T. (2009). ben Functions with scamp during synaptic transmission and long-term memory formation in Drosophila. J Neurosci 29, 414-424. 